\title{
Interior Point Algorithm for Multi-UAVs Formation Autonomous Reconfiguration
}

\author{
Wang Jian-hong ${ }^{1}$ and Rana Javed Masood ${ }^{2}$ \\ ${ }^{1}$ School of Mechanical and Electronic Engineering, Jingdezhen Ceramic Institute, Jingdezhen 333403, China \\ ${ }^{2}$ College of Automation Engineer, Nanjing University of Aeronautics and Astronautics, Nanjing, Jiangsu 210016, China \\ Correspondence should be addressed to Wang Jian-hong; wangjianhong@nuaa.edu.cn
}

Received 29 October 2015; Revised 25 May 2016; Accepted 28 June 2016

Academic Editor: Michela Robba

Copyright (c) 2016 W. Jian-hong and R. Javed Masood. This is an open access article distributed under the Creative Commons Attribution License, which permits unrestricted use, distribution, and reproduction in any medium, provided the original work is properly cited.

\begin{abstract}
Here the problem of designing multi-UAVs formation autonomous reconfiguration is considered. Combined with three kinds of cost functions, nonlinear dynamic equations, and four inequality constraints, one nonlinear multiobjective optimization problem is constructed. After applying weighted sum method and separating all equality or inequality constraints, the former nonlinear multiobjective optimization problem can be converted into a standard nonlinear single objective optimization problem. Then the interior point algorithm is applied to solve it. Further some improvements are proposed to avoid rank deficiency of some matrices. The equivalence property between multiobjective optimization and single objective optimization through weighted sum method is proved. Finally the efficiency of the proposed strategy can be confirmed by the simulation example results.
\end{abstract}

\section{Introduction}

Multi-UAVs formation is the basis and prerequisite for the UAV mission. When formation task or battlefield environment changes with time, the entire multi-UAVs formation needs to be adjusted. This adjustment is called formation autonomous reconfiguration. In reconfiguration process, each UAV adjusts its own position as a new geometry and plans the flight trajectory from the original location to the new terminal location. Thus the planned flight path ensures each UAV is secure while considering the nonlinear dynamics of UAV, various formations cost function, and a variety of constraints. So multi-UAVs formation autonomous reconfiguration is designed as a mathematical optimization problem in which decision variables are the control input sequences. The cost functions include three kinds of functions such as investigation UAV cost function, interference drone missile costs function, and radar jamming UAV cost function. Moreover the constraints include radar threat constraint, missile threat constraint, artillery positions constraint, and formations anticollision constraint.

An overview about the auxiliary role of the multi-UAVs formation trajectory design in a cooperative investigative process is given in [1]. In [2] an intelligent multiagents system is introduced and this multiagent system is represented as the communication structure between various UAVs. In [3] descriptions of many various methods of UAV path planning are summarized. In [4] the weights which exist in many intelligent algorithms are adjusted based on the gray system theory. In [5] the methods about how to fuse the multiple sensor information are analyzed to obtain state estimates from the perspective of information fusion. In [6] the particle filter algorithm is applied to track path under the nonGaussian condition. In [7] one interacting multiple model algorithm based on particle filter is proposed. In [8] the ant colony algorithm from the multiobjective optimization is studied. In [9] one consensus genetic algorithm is used to solve the multiobjective optimization problem. In [10] the use of game theory can transform the original multiobjective optimization problem into a Nash bargaining process. In [11] the communication delay is considered and one information compensated method based on the information filter algorithm is presented.

Here the interior point algorithm is applied in multiUAVs formation autonomous reconfiguration to design the autonomous trajectory. In the optimization model, three 
different cost functions are established and the dynamic equations of each UAV are regarded as equality constraints. After combining radar threats, missile threat, artillery positions threat, and formations anticollision, four kinds of inequality constraints are obtained. Based on these cost functions and equality and inequality constraints, an multiobjective optimization problem with equality and inequality constraints is constructed. The multiobjective optimization problem is solved to obtain the control input sequences which are used to plan trajectory. The main contribution of this paper is to analyze this multiobjective optimization problem by using operations algorithm. For this multiobjective optimization problem, the weighted sum strategy is applied to transform that multiobjective optimization into a single objective optimization and the feasibility of the weighted sum strategy is proved theoretically. In order to rewrite the original optimization problem into a standard form of nonlinear optimization, the kinetic equation for each equation UAV is further expanded and some forms are used to describe the set of state variables and control inputs. For the standard nonlinear optimization form, the major steps of the proposed interior point algorithm are given here. After calculating the optimal input sequences of nonlinear optimization problem by using the interior point algorithm, the idea of nonlinear receding horizon predictive control can be introduced. This idea is to choose the first term in the obtained optimal control input sequence and discard the remaining terms.

\section{Multi-UAVs Formation Autonomous Reconfiguration}

Multi-UAVs formation autonomous reconfiguration means that at initial time instant, multiple UAVs fly in a formation pattern. When the battlefield environment changes with time, the UAV swarm adjusts each UAV's former formation pattern and chooses one new flying pattern independently. In formation reconstruction processes, each UAV's position in the new formation mode requires to be redesigned in order to produce a new flight path. During the process of designing new flight path, the dynamics of each UAV, flight cost function, and constraints are considered. Multi-UAVs formation autonomous reconfiguration process is shown in Figure 1, where four different formation modes are described. It means that the groups of multiple UAVs sequentially select their own new formation modes according to the different surrounding environment.

Assume there are $n$ UAVs flying in formation, and the length of time is $N$. Since each UAV is decoupled with each other, this assumption means dynamic coupling phenomenon does not exist. Set state vector of the $i$ UAV as follows:

$$
x_{i}=\left[\begin{array}{llll}
x_{i}(1) & x_{i}(2) & \cdots & x_{i}(N)
\end{array}\right]^{T} .
$$

The control input sequence is that

$$
u_{i}=\left[\begin{array}{llll}
u_{i}(1) & u_{i}(2) & \cdots & u_{i}(N)
\end{array}\right]^{T} .
$$

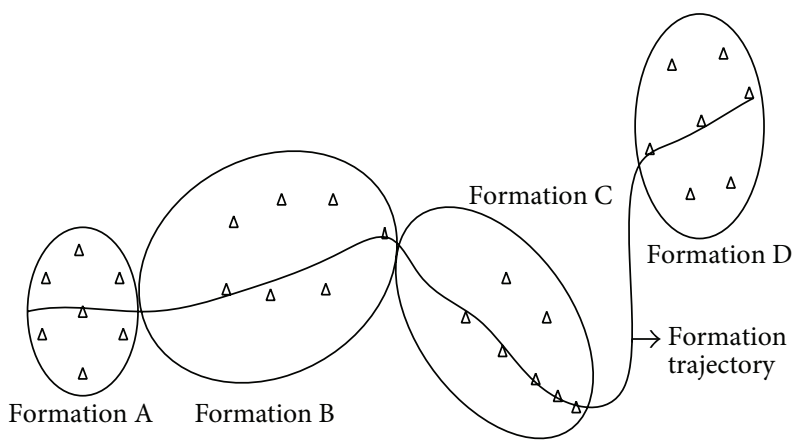

FIGURE 1: Multi-UAV formation autonomous reconfiguration process.

The time invariant discrete time state space equation is given as

$$
x_{i}(k+1)=f_{i}\left(x_{i}(k), u_{i}(k)\right) \quad x_{i} \in \Xi_{i}, u_{i}(k) \in \Theta_{i} .
$$

In (3), $f_{i}$ is a nonlinear mapping; this nonlinear mapping combines the state vector $x_{i}(k)$ at time $k$ and control input vector $u_{i}(k)$ to predict one new state variable $x_{i}(k+1)$ at the next time instant $k+1 ; \Xi_{i}$ and $\Theta_{i}$ are the feasible state set and control input constraint set with respect to the $i \mathrm{UAV}$.

The main contribution of this paper is to study how to transform the multiobjective optimization problem into a standard nonlinear optimization problem, and the classical interior point algorithm is applied to analyze the equivalence between the multiobjective optimization and single objective optimization by using weighted sum strategy.

When UAV formation leaps across the battlefield environment, there are many methods to define the cost function. For example, here define the position of the virtual lead aircraft at time $k$ as

$$
p_{l}(k)=\left[\begin{array}{ll}
x_{l}(k) & y_{l}(k)
\end{array}\right]^{T} .
$$

The position of $i$ UAV $v_{i}$ whose role is to carry out the reconnaissance mission is denoted as

$$
p_{i}(k)=\left[\begin{array}{ll}
x_{i}(k) & y_{i}(k)
\end{array}\right]^{T} .
$$

The cost function corresponding to UAV $v_{i}$ during the whole reconnaissance mission is defined as

$$
F_{i}^{1}\left(x_{i}, u_{i}\right)=\sum_{k=1}^{N}\left(\left\|\left(p_{l}(k)-p_{i}(k)\right)^{2}+\right\| u_{i}(k)\left\|_{R_{i}}^{2}\right\|\right) \text {. }
$$

In (6), $R_{i}$ represents a positive definite weighting matrix; the second term is used to normalize the original optimization problem and ensure that the optimal solution will not depart the true solution. Similarly two other cost functions coming from the missile and radar interference are given:

$$
\begin{aligned}
& F_{i}^{2}\left(x_{i}, u_{i}\right)=\sum_{k=1}^{N}\left(\left\|\left(p_{a_{1}}(k)-p_{i}(k)\right)^{2}+\right\| u_{i}(k)\left\|_{R_{i}}^{2}\right\|\right) \\
& F_{i}^{3}\left(x_{i}, u_{i}\right)=\sum_{k=1}^{N}\left(\left\|\left(p_{a_{2}}(k)-p_{i}(k)\right)^{2}+\right\| u_{i}(k)\left\|_{R_{i}}^{2}\right\|\right) .
\end{aligned}
$$


In (7), the cost function $F_{i}^{2}\left(x_{i}, u_{i}\right)$ is constructed by the distance between the current position $p_{a_{1}}$ and ideal position $p_{a_{1}}$ of the missile interference that interferes the $i$ UAV. $p_{a_{1}}$ is the ideal position of the missile interference. In (8), the cost function $F_{i}^{3}\left(x_{i}, u_{i}\right)$ is constructed by the distance between the current position $p_{i}$ and ideal position $p_{a_{2}}$ of the radar interference that interferes the $i \mathrm{UAV}$. This cost function $F_{i}^{3}\left(x_{i}, u_{i}\right)$ can achieve the maximum protection under formation flight path.

In total there are $n$ UAVs lying in the formation geometry. Combining (6), (7), and (8), we consider the following cost function:

$$
\begin{aligned}
& \min _{u}\left[F_{i}^{1}\left(x_{i}, u_{i}\right) \quad F_{i}^{2}\left(x_{i}, u_{i}\right) \quad F_{i}^{3}\left(x_{i}, u_{i}\right)\right]_{i=1}^{n} \\
& u=\left[\begin{array}{llll}
u_{1} & u_{2} & \cdots & u_{n}
\end{array}\right]^{T} .
\end{aligned}
$$

In (9), the total number of elements in the minimization operation is $3 n$. The minimum solution $u$ which guarantees all $3 n$ elements can achieve their own minimization simultaneously does not exist. Fortunately one compromise solution can be found and this compromise solution is called the efficient solution in multiobjective optimization theory. Under not any constraints, the optimization problem (9) can be solved by the weighted sum strategy. But in multi-UAVs formation autonomous reconfiguration problem, four kinds of inequality constraints are considered; the interior point algorithm can only be used to get an efficient solution.

All kinds of constraints include the radar threat constraint, missile threat constraint, artillery positions threat, and formations anticollision constraint. Their respective inequalities are defined as follows in turn.

Setting the position and detection radius of the radar $r_{j}$ as $p_{r_{j}}=\left[\begin{array}{ll}x_{r_{j}} & y_{r_{j}}\end{array}\right]^{T}$ and $R_{r_{j}}(k)$, respectively, then the radar threat constraint corresponding to the UAV $v_{i}$ is defined as follows:

$$
g_{i}^{1}\left(x_{i}(k), u_{i}(k)\right)=R_{r_{j}}(k)^{2}-\left\|p_{i}(k)-p_{r_{j}}(k)\right\|^{2} \leq 0 .
$$

Setting the position of missile $m_{j}$ as $p_{m_{j}}=\left[\begin{array}{ll}x_{m_{j}} & y_{m_{j}}\end{array}\right]^{T}$, the safety distance and safety angle cosine after disturbing missile $m_{j}$ are $d_{m_{j}}$ and $c s_{m_{j}}(k)$, respectively. Then missile threat constraint corresponding to the UAV $v_{i}$ is defined as follows:

$$
g_{i}^{2}\left(x_{i}(k), u_{i}(k)\right)=c s_{m_{j}}(k)-\cos \left(\frac{\theta}{2}\right) \leq 0 .
$$

Assuming that the radiation radius of artillery positions is not influenced by external disturbance, we set this radiation radius as a constant. The position and radiation radius of artillery $n_{j}$ are defined as $p_{n_{j}}=\left[\begin{array}{ll}x_{n_{j}} & y_{n_{j}}\end{array}\right]^{T}$ and $R_{n_{j}}(k)$, respectively; then artillery positions threat corresponding to the $\mathrm{UAV} v_{i}$ is defined as follows:

$$
g_{i}^{3}\left(x_{i}(k), u_{i}(k)\right)=R_{n_{j}}(k)^{2}-\left\|p_{i}(k)-p_{n_{j}}(k)\right\|^{2} \leq 0 .
$$

The minimum safe distance among UAV formation is denoted by $d_{\text {min }}$, and then formations anticollision constraint corresponding to the UAV $v_{i}$ is defined as follows:

$$
g_{i}^{4}\left(x_{i}(k), u_{i}(k)\right)=d_{\min }^{2}-\left\|p_{i}(k)-p_{j}(k)\right\|^{2} \leq 0 .
$$

Unifying the above multiobjective function (9), nonlinear dynamical equations (3), and four inequality constraints (10)(13), multi-UAVs formation autonomous reconfiguration problem can be formulated as a nonlinear multiobjective optimization problem:

$$
\min _{u}\left[F_{i}^{1}\left(x_{i}, u_{i}\right) F_{i}^{2}\left(x_{i}, u_{i}\right) F_{i}^{3}\left(x_{i}, u_{i}\right)\right]_{i=1}^{n}
$$

subject to $x_{i}(k+1)=f_{i}\left(x_{i}(k), u_{i}(k)\right)$

$$
\begin{aligned}
& x_{i} \in \Xi_{i}, \\
& u_{i}(k) \in \Theta_{i} \\
& i=1,2, \ldots, \\
& g_{i}^{1}\left(x_{i}(k), u_{i}(k)\right) \leq 0, \\
& g_{i}^{2}\left(x_{i}(k), u_{i}(k)\right) \leq 0, \\
& g_{i}^{3}\left(x_{i}(k), u_{i}(k)\right) \leq 0, \\
& g_{i}^{4}\left(x_{i}(k), u_{i}(k)\right) \leq 0 .
\end{aligned}
$$$$
i=1,2, \ldots, n, k=1,2, \ldots, N
$$

Combining all the inequality constraints in (14) and vectoring them, we get

$$
\begin{gathered}
g_{i}(x, u)=\left[\begin{array}{c}
g_{i}^{1}\left(x_{i}(k), u_{i}(k)\right) \\
g_{i}^{2}\left(x_{i}(k), u_{i}(k)\right) \\
g_{i}^{3}\left(x_{i}(k), u_{i}(k)\right) \\
g_{i}^{4}\left(x_{i}(k), u_{i}(k)\right)
\end{array}\right], \\
g(x, u)=\left[\begin{array}{c}
g_{1}(x, u) \\
\vdots \\
g_{n}(x, u)
\end{array}\right] \leq 0,
\end{gathered}
$$

where the state variable and control input vector are given, respectively, as follows:

$$
\begin{aligned}
& x=\left[\begin{array}{llll}
x_{1} & x_{2} & \cdots & x_{n}
\end{array}\right]^{T}, \\
& u=\left[\begin{array}{llll}
u_{1} & u_{2} & \cdots & u_{n}
\end{array}\right]^{T} .
\end{aligned}
$$

Similarly combining all the nonlinear dynamics equations of each UAV, we get

$$
\begin{aligned}
x(k+1) & =\left[\begin{array}{c}
x_{1}(k+1) \\
x_{2}(k+1) \\
\vdots \\
x_{n}(k+1)
\end{array}\right]=\left[\begin{array}{c}
f_{1}\left(x_{1}(k), u_{1}(k)\right) \\
f_{2}\left(x_{2}(k), u_{2}(k)\right) \\
\vdots \\
f_{n}\left(x_{n}(k), u_{n}(k)\right)
\end{array}\right] \\
& =f(x(k), u(k)) .
\end{aligned}
$$


Using the vector form (15), (16), and (17), nonlinear multiobjective optimization problem can be simplified as

$$
\min _{u}\left[F_{i}^{1}\left(x_{i}, u_{i}\right) F_{i}^{2}\left(x_{i}, u_{i}\right) F_{i}^{3}\left(x_{i}, u_{i}\right)\right]_{i=1}^{n}
$$

subject to $x(k+1)=f(x(k), u(k))$

$$
\begin{aligned}
& x_{i} \in \Xi_{i}, \\
& u_{i}(k) \in \Theta_{i} \\
& \qquad i=1,2, \ldots, n, k=1,2, \ldots, N \\
& g(x, u) \leq 0 .
\end{aligned}
$$

Before solving (18) by the interior point algorithm, we transform the multiobjective optimization problem (18) into a nonlinear optimization problem with equality and inequality constraints.

\section{Standardized Model}

In (18), the cost function is a multiobjective vector:

$$
\min _{u}\left[F_{i}^{1}\left(x_{i}, u_{i}\right) F_{i}^{2}\left(x_{i}, u_{i}\right) F_{i}^{3}\left(x_{i}, u_{i}\right)\right]_{i=1}^{n} .
$$

Applying the weighting sum strategy and transforming to one single objective optimization problem,

$$
\begin{array}{rl}
\min _{u} & F(u) \\
& =\sum_{i=1}^{n}\left[\lambda_{1 i} F_{i}^{1}\left(x_{i}, u_{i}\right)+\lambda_{2 i} F_{i}^{2}\left(x_{i}, u_{i}\right)+\lambda_{3 i} F_{i}^{3}\left(x_{i}, u_{i}\right)\right] .
\end{array}
$$

In (20), vector $\left\{\lambda_{1 i}, \lambda_{2 i}, \lambda_{3 i}\right\}_{i=1}^{n}$ indicates positive weighted scalar values and the following conditions are satisfied:

$$
\sum_{i=1}^{n}\left[\lambda_{1 i}+\lambda_{2 i}+\lambda_{3 i}\right]=1 \quad \lambda_{1 i}>0, \lambda_{2 i}>0, \lambda_{3 i}>0
$$

The nonlinear dynamic equations of UAV (18) can be rewritten as follows:

$$
\begin{aligned}
x_{0}-x_{0} & =0 \\
x(k+1)-f(x(k), u(k)) & =0, \quad k=1,2 \cdots N-1 .
\end{aligned}
$$

In (22), the first equation represents the initial state. For the set of feasible state $\Xi_{i}$ and control input constraint set $\Theta_{i}$, define the following constraints:

$$
\begin{aligned}
\Xi_{i} & =\left\{\frac{x_{i}}{\exists u_{i}} \in \Theta_{i}, \text { with } G_{i}^{s}\left(x_{i}, u_{i}\right)=0, H_{i}^{s}\left(x_{i}, u_{i}\right)\right. \\
& \leq 0\} \\
\Theta_{i} & =\left\{\frac{u_{i}}{\exists x_{i}} \in \Xi_{i}, \text { with } G_{i}^{s}\left(x_{i}, u_{i}\right)=0, H_{i}^{s}\left(x_{i}, u_{i}\right)\right. \\
& \leq 0\} .
\end{aligned}
$$

The two above functions $G_{i}^{s}\left(x_{i}, u_{i}\right)$ and $H_{i}^{s}\left(x_{i}, u_{i}\right)$ do not depend on two variables $x_{i}$ and $u_{i}$ simultaneously.

Combining all the equations and inequalities together and using (20), we obtain a standard nonlinear optimization problem:

$$
\begin{aligned}
& \min _{u} F(u)=\sum_{i=1}^{n}\left[\lambda_{1 i} F_{i}^{1}\left(x_{i}, u_{i}\right)+\lambda_{2 i} F_{i}^{2}\left(x_{i}, u_{i}\right)+\lambda_{3 i} F_{i}^{3}\left(x_{i}, u_{i}\right)\right] \\
& \text { with respect to } u=\left[\begin{array}{llll}
u_{1} & u_{2} & \cdots & u_{n}
\end{array}\right]^{T} \\
& \text { subject to } G(u)=\left[\begin{array}{c}
x_{0}-x_{0} \\
{[x(k+1)-f(x(k), u(k))]_{k=1}^{N-1}} \\
{\left[G_{i}^{s}\left(x_{i}, u_{i}\right)\right]_{i=1}^{n}}
\end{array}\right]=0 \text {, } \\
& H(u)=\left[\begin{array}{c}
g(x, u) \\
{\left[H_{i}^{s}\left(x_{i}, u_{i}\right)\right]_{i=1}^{n}}
\end{array}\right] \leq 0 .
\end{aligned}
$$

In order to solve the standard nonlinear optimization problem (20), the interior point algorithm is proposed.

\section{Interior Point Algorithm}

The purpose of the interior point algorithm is to generate an iterative sequence $u^{j}$; here the superscript symbol $u^{j}$ is different with control input $u(k)$ at time instant $k$. This generated sequence will be included in the control input set. In the iteration process of each generating sequence, each element of the inequality constraint $H(u)$ is considered. After introducing the slack variable $s$, those inequality constraints can be converted into equality constraints [12]. The standard nonlinear optimization problem (24) can be rewritten as

$$
\begin{aligned}
& \min _{u} \quad F(u) \\
& \text { subject to } \quad G(u)=0, \\
& H(u)+s=0, \quad s \geq 0 .
\end{aligned}
$$


In (25), the slack variable $s$ is chosen as a vector with appropriate dimension, and each of its elements is nonnegative. Then we construct one Lagrangian function corresponding to (25) as follows:

$$
L(u, s, v, w)=F(u)-v^{T} G(u)-w^{T}(H(u)+s) .
$$

According to the necessary condition from the optimality theory, the equation $H(u)+s=0$ holds at the minimum value.

Applying the optimality Karush-Kuhn-Tucker sufficient and necessary condition [13], we obtain

$$
\begin{aligned}
\nabla F(u)-\nabla G^{T}(u) v-\nabla H^{T}(u) w & =0 \\
S w-\mu e & =0 \\
G(u) & =0, \\
H(u)+s & =0 .
\end{aligned}
$$

From (27), we have that

$$
\begin{aligned}
s & \geq 0, \\
w & \geq 0, \\
\mu & =0, \\
e & =\left(\begin{array}{llll}
1 & 1 & \cdots & 1
\end{array}\right)^{T} .
\end{aligned}
$$

In (27), define the following two matrices:

$$
\begin{gathered}
S=\operatorname{diag}(s), \\
W=\operatorname{diag}(w) .
\end{gathered}
$$

In (27), the introduction of perturbation parameter $\mu$ is to guarantee that if we choose $\mu>0$, the iteration sequence will be far away from the boundary of the control input set. The choice of the perturbation parameters $\mu$ satisfies that

$$
\mu_{j} \longrightarrow 0 \quad \text { as } j \longrightarrow \infty \text {. }
$$

In order to improve the performance of the optimal conditions (27), we use the interior point algorithm to solve (25) and introduce barrier function to eliminate the nonnegative condition $s \geq 0$ :

$$
\begin{aligned}
& \min _{u} F(u)-\mu \sum_{i=1}^{n_{s}} \log s_{i} \\
& \text { subject to } \mathrm{G}(u)=0 \text {, } \\
& H(u)+s=0 . \\
& \nabla F(u)-\nabla G^{T}(u) v-\nabla H^{T}(u) w=0 \\
& \mu S^{-1} e+w=0 \\
& G(u)=0, \\
& H(u)+s=0 .
\end{aligned}
$$

The barrier function $-\mu \sum_{i=1}^{n_{s}} \log s_{i}$ is added here to prevent each element of slack variable $s$ to approach 0 closely. Also applying the generalized optimality KKT sufficient and necessary condition [14] in (31) with one barrier function, we obtain
Using Newton increment steps in (32), we obtain the following system equation:

$$
\begin{gathered}
{\left[\begin{array}{cccc}
\nabla_{u u}^{2} L(u, s, v, w) & 0 & -\nabla G^{T}(u) & -\nabla H^{T}(u) \\
0 & W & 0 & S \\
\nabla G(u) & 0 & 0 & 0 \\
\nabla H(u) & -I & 0 & 0
\end{array}\right]\left[\begin{array}{c}
\Delta u \\
\Delta s \\
\Delta v \\
\Delta w
\end{array}\right]} \\
=-\left[\begin{array}{c}
\nabla F(u)-\nabla G^{T}(u) v-\nabla H^{T}(u) w \\
S w-\mu e \\
G(u) \\
H(u)+s
\end{array}\right] .
\end{gathered}
$$

After calculating the increment,

$$
\left[\begin{array}{llll}
\Delta u & \Delta s & \Delta v & \Delta w
\end{array}\right]
$$

The new recursive iteration values are calculated as

$$
\begin{gathered}
u^{j+1}=u^{j}+\alpha_{s}^{\max } \Delta u, \\
s^{j+1}=s^{j}+\alpha_{s}^{\max } \Delta s \\
v^{j+1}=v^{j}+\alpha_{w}^{\max } \Delta v, \\
w^{j+1}=w^{j}+\alpha_{w}^{\max } \Delta w,
\end{gathered}
$$

where two steps $\alpha_{s}^{\max }$ and $\alpha_{w}^{\max }$ are chosen as

$$
\begin{aligned}
& \alpha_{s}^{\max } \\
& =\max \{\alpha \in(0,1) \text { such that } s+\alpha \Delta s \geq(1-\tau) s\} \\
& \alpha_{w}^{\max } \\
& =\max \{w \in(0,1) \text { such that } w+\alpha \Delta w \geq(1-\tau) w\},
\end{aligned}
$$

where $\tau \in(0,1)$, and take $\tau=0.995$. To ensure the second block matrix in (33) has full row rank and this matrix is not singular, we rewrite (33) as follows:

$$
\begin{gathered}
{\left[\begin{array}{cccc}
\nabla_{u u}^{2} L(u, s, v, w) & 0 & -\nabla G^{T}(u) & -\nabla H^{T}(u) \\
0 & \sum & 0 & I \\
-\nabla G(u) & 0 & 0 & 0 \\
-\nabla H(u) & I & 0 & 0
\end{array}\right]\left[\begin{array}{c}
\Delta u \\
\Delta s \\
\Delta v \\
\Delta w
\end{array}\right]} \\
=-\left[\begin{array}{c}
\nabla F(u)-\nabla G^{T}(u) v-\nabla H^{T}(u) w \\
w-\mu S^{-1} e \\
-G(u) \\
-H(u)-s
\end{array}\right],
\end{gathered}
$$

where $\Sigma=S^{-1} W$, as in the iterative process, the normed function is a decreasing function. It means that the incremental vector $\left[\begin{array}{llll}\Delta u & \Delta s & \Delta v & \Delta w\end{array}\right]$ is a descent direction, so it is desirable to choose matrix

$$
\left[\begin{array}{cc}
\nabla_{u u}^{2} L(u, s, v, w) & 0 \\
0 & \Sigma
\end{array}\right]
$$


as a positive definite matrix which is on the null space with respect to matrix

$$
\left(\begin{array}{cc}
-\nabla G(u) & 0 \\
-\nabla H(u) & I
\end{array}\right)
$$

From the construction of $\sum$, it means $\sum$ is a positive definite matrix. But Hessian matrix $\nabla_{u u}^{2} L(u, s, v, w)$ may be negative definite. To compensate for this defection, we use $\nabla_{u u}^{2} L(u, s, v, w)+\delta I$ to replace Hessian matrix $\nabla_{u u}^{2} L(u, s, v, w)$. Scalar $\delta>0$ is chosen sufficiently large to ensure the positive definiteness of the Hessian matrix. Additionally in the interior point algorithm, the rank deficiency of the gradient matrix $\nabla G(u)$ is considered. So we make the following modifications to the primal-dual matrices:

$$
\begin{gathered}
{\left[\begin{array}{cccc}
\nabla_{u u}^{2} L(u, s, v, w)+\delta I & 0 & -\nabla G^{T}(u) & -\nabla H^{T}(u) \\
0 & \sum & 0 & I \\
-\nabla G(u) & 0 & \gamma I & 0 \\
-\nabla H(u) & I & 0 & 0
\end{array}\right]\left[\begin{array}{c}
\Delta u \\
\Delta s \\
\Delta v \\
\Delta w
\end{array}\right]} \\
=-\left[\begin{array}{c}
\nabla F(u)-\nabla G^{T}(u) v-\nabla H^{T}(u) w \\
w-\mu S^{-1} e \\
-G(u) \\
-H(u)-s
\end{array}\right] .
\end{gathered}
$$

One normalized parameter $\gamma>0$ is added in the first matrix (40). As the iterative expressions (35) do not terminate in a limited period of time, one error criterion function can be applied to determine when to stop the entire iterative algorithm. This error criterion function may be that

$$
\begin{aligned}
& E(u, s, v, w) \\
& \quad=\max \left\{\left\|\nabla F(u)-\nabla G^{T}(u) v-\nabla H^{T}(u) w\right\|,\right. \\
& \|S w-\mu e\|,\|G(u)\|,\|H(u)+s\|\} .
\end{aligned}
$$

With this error criterion function as a judge to terminate the iterative algorithm, we summarize the basic steps of the interior point algorithm.

Step 1. Assume a pair of initial value is given as $\left(u^{0}, s^{0}\right)$, and let $j=0$.

Step 2. Calculate Lagrange multipliers $v^{0}$ and $w^{0}$ and define the parameters

$$
\begin{gathered}
\mu^{0}>0, \\
\sigma, \tau \in(0,1) .
\end{gathered}
$$

Step 3. Verify whether the error criterion function holds:

$$
E\left(u^{j}, s^{j}, v^{j}, w^{j}\right) \geq \varepsilon .
$$

$\varepsilon$ is a very small positive number; if above equality does not hold, then stop the iterative algorithm. Then the optimization variables $\left(u^{j}, s^{j}, v^{j}, w^{j}\right)$ at this time can be regarded as the optimal solution to a nonlinear optimization problem.
Step 4. Solve (40) and calculate the search direction $\left[\begin{array}{llll}\Delta u & \Delta s & \Delta v & \Delta w\end{array}\right]$.

Step 5. Use (36) to determine two steps $\alpha_{s}^{\max }$ and $\alpha_{w}^{\max }$.

Step 6. Use the iterative expression (35) to solve the new iterative value.

Step 7. Let $\mu^{j+1}=\mu^{j}, k=k+1$.

Step 8. Return to Step 3.

In above steps, we make some modifications to compensate the drawback of the usual interior point algorithm. These modifications can guarantee the optimal solution of the original nonlinear optimization problem [15].

\section{Equivalence between the Two Optimization Problems}

When solving the multiobjective optimization problem, firstly we apply the weighted sum strategy to convert it into one single objective optimization problem. Under the conditions of the positive weighted scalars (21), the equivalence between these two optimization problems holds. As there are $3 n$ elements in (19), rewrite equality (19) as follows:

$$
\min _{u}\left[\begin{array}{lllll}
F_{1}(u) & F_{2}(u) & \cdots & F_{3 n}(u)
\end{array}\right] .
$$

Similarly rewrite equality (20) as follows:

$$
\min _{u} F(u)=\sum_{i=1}^{3 n} \lambda_{i} F_{i}(u) .
$$

Using (44) and (45), the conditions of equivalence between (44) and (45) can be summarized as the following proposition.

Proposition 1. Let $\lambda_{i}>0, i=1,2, \ldots, 3 n$, and $\sum_{i=1}^{3 n} \lambda_{i}=1$. If $\widehat{u}$ is an optimal solution of (45), then $\widehat{u}$ is an effective solution of (44).

Proof. Let $\widehat{u}$ be an optimal solution of (45), and it satisfies $F\left(u^{\prime}\right) \leq F(\widehat{u})$. Applying the use of positive weights $F_{i}\left(u^{\prime}\right) \leq$ $F_{i}(\widehat{u}), i=1,2 \cdots 3 n$, we obtain the following equation:

$$
\sum_{i=1}^{3 n} \lambda_{i} F_{i}\left(u^{\prime}\right)<\sum_{i=1}^{3 n} \lambda_{i} F_{i}(\widehat{u}) .
$$

Choose a sufficiently large positive number $M$ such that the negative result about optimal solution $\widehat{u}$ holds. This positive number $M$ is chosen as

$$
M=(3 n-1) \max _{i, j} \frac{\lambda_{j}}{\lambda_{i}} .
$$

Next we use the contradiction method to prove this proposition. Let $\widehat{u}$ be not an effective solution of (44), then there exists one $i \in\{1,2 \cdots 3 n\}$ and $u$ such that

$$
\begin{gathered}
F_{i}(u) \leq F_{i}(\widehat{u}) \\
F_{i}(\widehat{u})-F_{i}(u)>M\left(F_{i}(u)-F_{i}(\widehat{u})\right) .
\end{gathered}
$$


For all $j \in\{1,2 \cdots 3 n\}$, we have

$$
F_{j}(u) \geq F_{j}(\widehat{u}) .
$$

Continuing to formulate, we get

$$
F_{i}(\widehat{u})-F_{i}(u)>\frac{3 n-1}{\lambda_{i}} \lambda_{j}\left(F_{j}(u)-F_{j}(\widehat{u})\right), \quad j \neq i .
$$

Multiplying both sides of (50) by $\lambda_{i} /(3 n-1)$ and taking sum operations, we have

$$
\begin{gathered}
\lambda_{i}\left(F_{i}(\widehat{u})-F_{i}(u)\right)>\sum_{j \neq i} \lambda_{j}\left(F_{j}(u)-F_{j}(\widehat{u})\right) \Longleftrightarrow \\
\lambda_{i} F_{i}(\widehat{u})-\lambda_{i} F_{i}(u)>\sum_{j \neq i} \lambda_{j} F_{j}(u)-\sum_{j \neq i} \lambda_{j} F_{j}(\widehat{u}) \Longleftrightarrow \\
\lambda_{i} F_{i}(\widehat{u})+\sum_{j \neq i} \lambda_{j} F_{j}(\widehat{u})>\lambda_{i} F_{i}(u)+\sum_{j \neq i} \lambda_{j} F_{j}(u) \Longleftrightarrow \\
\sum_{i=1}^{3 n} \lambda_{i} F_{i}(u)<\sum_{i=1}^{3 n} \lambda_{i} F_{i}(\widehat{u}) .
\end{gathered}
$$

In (51), the last inequality contradicts the assumption that $\widehat{u}$ is an optimal solution of (45). So it means that $\widehat{u}$ is an effective solution of (44).

\section{Numerical Example}

To verify the interior point algorithm in multi-UAVs formation autonomous reconfiguration, the formation includes three UAVs: one radar jamming UAV, one missile jamming UAV, and one investigation UAV. The initial positions of three UAVs are all located at starting point coordinates $(0,0)$ and the terminate positions are concentrated on coordinates $(700,700)$. The vector of the flight maximum speed, minimum speed, and the speed deviation is that

$$
\left[\begin{array}{lll}
80 & 15 & \pm 5
\end{array}\right] \text {. }
$$

Surrounding battlefield environment contains radar threat, missile threat, and antiaircraft positions threat. The deployment coordinates of the radar threat are $(300,300)$, and the deployment coordinates of the missile threat are $(250,200)$. The region of the artillery positions threat is a rectangle range with a height of 300 and a width of 300 . This rectangle range belongs to the no-fly zone.

Applying the interior point algorithm in multi-UAVs formation autonomous reconfiguration, the weighted factor for each UAV is

$$
Q_{i}=\operatorname{diag}\{10,10\}
$$

The weighted matrix in the cost function is

$$
R_{i}=\operatorname{diag}\{1,10\} .
$$

The discrete time sampling period is taken as

$$
\Delta T=0.05 \text { s. }
$$

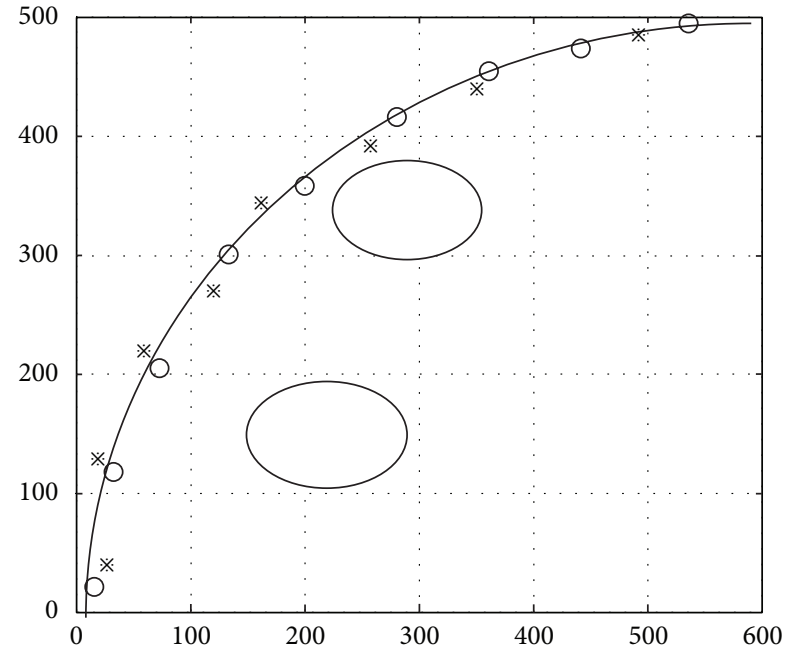

FIGURE 2: Multi-UAV formation autonomous reconfiguration trajectory.

The length of time is $N=500 \mathrm{~s}$, the number of UAVs is $n=$ 3 , and the initial value during the interior point algorithm is chosen as follows:

$$
\left(\begin{array}{lll}
u^{0}, s^{0}
\end{array}\right)=\left(\begin{array}{llll}
0.01 & 0.01 & 0.01 & 0.01
\end{array}\right)^{T} .
$$

Perturbation parameter is $\mu^{0}=0.05$, a positive number is $\varepsilon=0.01$, the normalized parameter is $\gamma=0.5$, and the scalar is $\delta=1.5$.

The simulation trajectory is shown in Figure 2. The coordinates of threat in surrounding battlefield environment are set as $(296,346)$ and $(229,173)$; the effect radius of the threat is $100 \mathrm{~m}$. Assume the threat of the threat zero is infinite. In order to increase the flight performance, the density of sampling points in the vicinity of the threat can be higher than the area of no threat. In Figure 2, $\bigcirc$ denotes the global optimal navigation point and $※$ denotes the position of the optimal navigation point. Two annular regions represent a range of threats.

From Figure 2, three UAVs formations experience a total of three formation autonomous reconfiguration processes. The first autonomous reconfiguration occurs in coordinates $(200,100)$; the second occurs in coordinates $(280,280)$. After these two formation autonomous reconfigurations, three UAVs can bypass these three threats and fly from initial position to terminate position.

Figure 3 shows the iterative convergence curves of cost functions with respect to three UAVs. Here each cost function is written as formula (20) with the weighted scalar value being $\lambda=1 / 9$. From Figure 3, with the interior point algorithm runs, after we substitute the optimal control input solution $u$ into the corresponding weighted sum cost function, the cost function will approach to zero closely with increasing number of iteration steps.

\section{Conclusion}

After establishing one nonlinear multiobjective optimization problem in multi-UAVs formation autonomous reconfiguration, we use weighted sum strategy and combine all the 


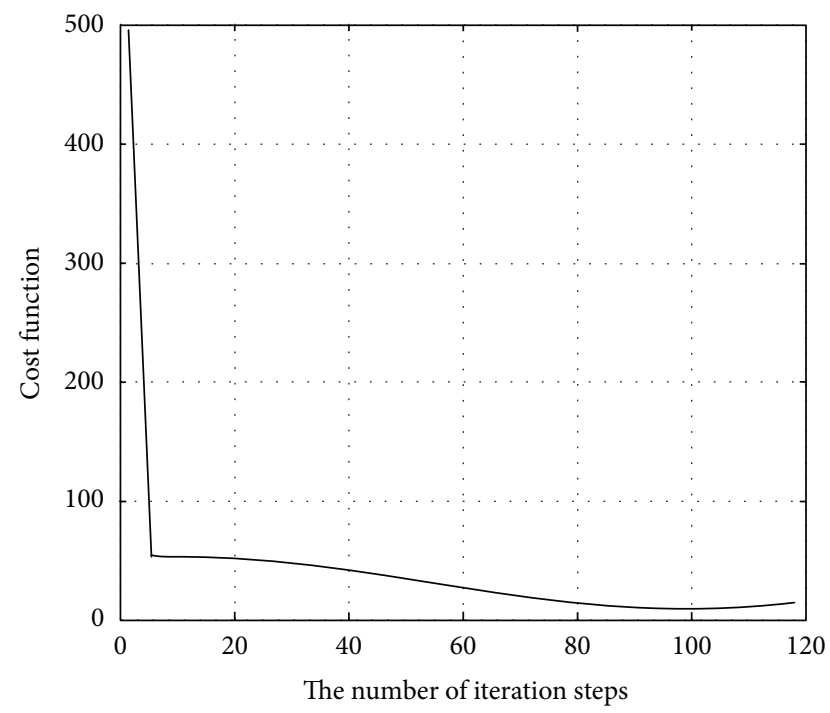

(a) UAV1

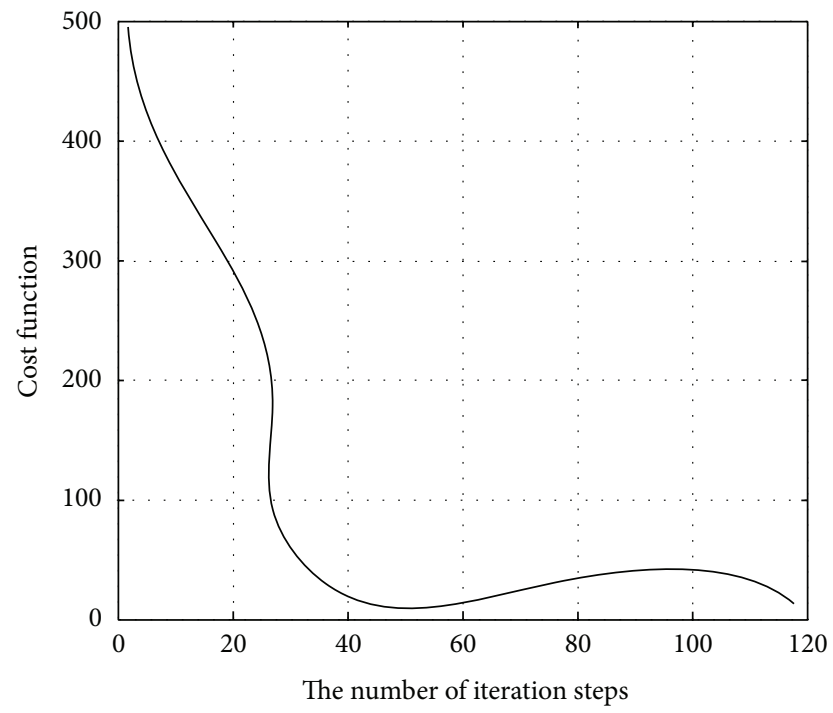

(b) UAV2

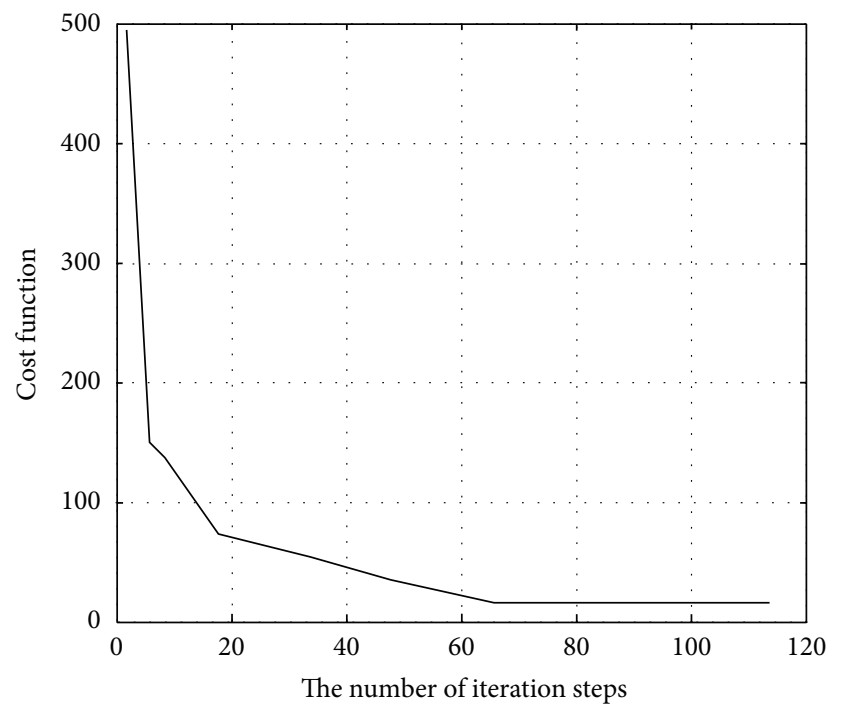

(c) UAV3

FIGURE 3: Convergence curves of each UAV cost function.

equations or inequalities to derive a standard single objective nonlinear optimization problem. Furthermore the interior point algorithm is proposed to solve the optimization problem and some improvements are made to ensure the optimal solution of the original nonlinear optimization problem. But in this paper, the asymptotic property and sensitivity analysis are not studied, so these two are the next research subjects.

\section{Competing Interests}

The authors declare that there is no conflict of interests regarding the publication of this paper.

\section{Acknowledgments}

This work was supported by the Grants from the National Science Foundation of China (no. 31260273), the ChinaMontenegro Intergovernmental S\&T Cooperation, and the
JiangXI Provincial Foundation for Leaders of Disciplines in Science (20113BCB22008).

\section{References}

[1] A. Karimoddini, H. Lin, B. M. Chen, and T. H. Lee, "Hybrid three-dimensional formation control for unmanned helicopters," Automatica, vol. 49, no. 2, pp. 424-433, 2013.

[2] M. Innocenti, L. Pollini, and F. Giulietti, "Management of communication failures in formation flight," Journal of Aerospace Computing, Information and Communication, vol. 11, no. 1, pp. 19-35, 2004.

[3] H. Rezaee and F. Abdollahi, "Motion synchronization in unmanned aircrafts formation control with communication delays," Communications in Nonlinear Science and Numerical Simulation, vol. 18, no. 3, pp. 744-756, 2013.

[4] B. I. Triplett, D. J. Klein, and K. A. Morgansen, "Cooperative estimation for coordinated target tracking in a cluttered 
environment," Mobile Networks and Applications, vol. 14, no. 3, pp. 336-349, 2009.

[5] S. Y. Li, Y. Zhang, and Q. Zhu, "Nash-optimization enhanced distributed model predictive control applied to the Shell benchmark problem," Information Sciences, vol. 170, no. 2-4, pp. 329349, 2005.

[6] D. Schoerling, C. Van Kleeck, F. Fahimi, C. R. Koch, A. Ams, and P. Löber, "Experimental test of a robust formation controller for marine unmanned surface vessels," Autonomous Robots, vol. 28, no. 2, pp. 213-230, 2010.

[7] H. Sun, R. Zhou, L. Zou, and Q. Ding, "Distributed cooperation target tracking for heterogeneous multi-UAV under communication and measurement constraints," Acta Aeronautica et Astronautica Sinica, vol. 32, no. 2, pp. 299-310, 2011.

[8] X.-Q. Zhang, "Research on improved leader following formation method," Computer Engineering and Design, vol. 31, no. 11, pp. 2547-2549, 2010.

[9] F. Giulietti, L. Pollini, and M. Innocenti, "Autonomous formation flight," IEEE Control Systems Magazine, vol. 20, no. 6, pp. 34-44, 2000.

[10] R. C. Jian and R.-X. Wei, "Nash bargaining based selfreconfigurable UAV formation control method," Automatica Sinica, vol. 39, no. 8, pp. 1349-1359, 2013.

[11] R. C. Jian and R.-X. Wei, "UAV under communication delay conditions formation of independent security control reconstruction," Control Theory \& Applications, vol. 30, no. 9, pp. 1099-1108, 2013.

[12] F. Giulietti, M. Innocenti, M. Napolitano, and L. Pollini, "Dynamic and control issues of formation flight," Aerospace Science and Technology, vol. 9, no. 1, pp. 65-71, 2005.

[13] Y. Ben-Asher, S. Feldman, P. Gurfil, and M. Feldman, "Distributed decision and control for cooperative UAVs Using Ad Hoc communication," IEEE Transactions on Control Systems Technology, vol. 16, no. 3, pp. 511-516, 2008.

[14] W.-H. Chen, "Nonlinear disturbance observer-enhanced dynamic inversion control of missiles," Journal of Guidance, Control, and Dynamics, vol. 26, no. 1, pp. 161-166, 2003.

[15] F. Giulietti, "Dynamics and control of different aircraft formation structures," Aeronautical, vol. 108, no. 10, pp. 117-124, 2004. 


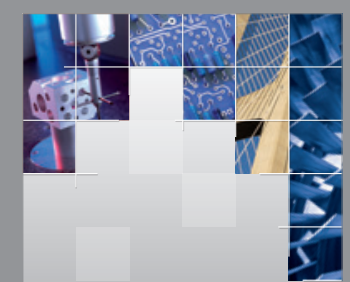

\section{Enfincering}
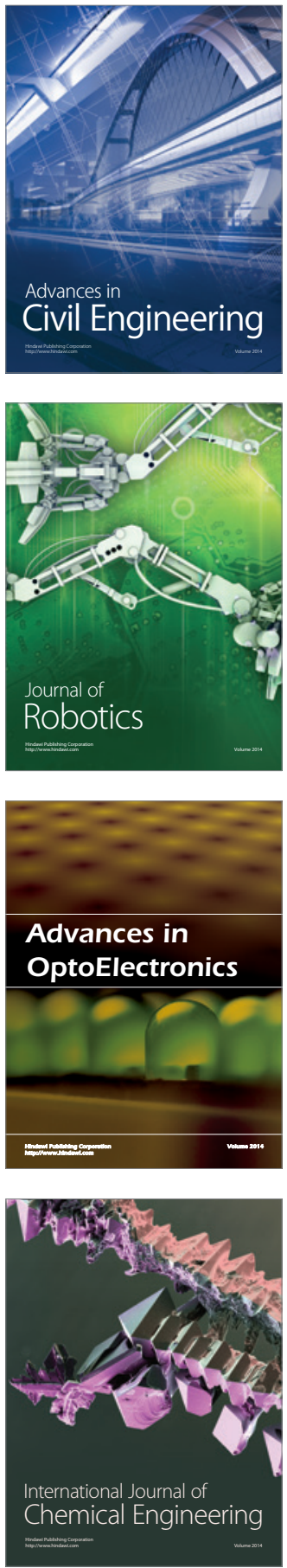

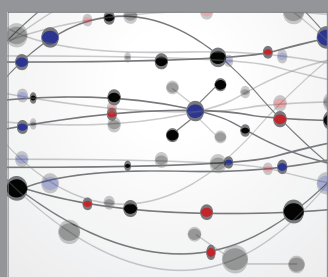

The Scientific World Journal

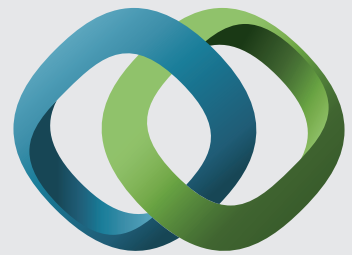

\section{Hindawi}

Submit your manuscripts at

http://www.hindawi.com
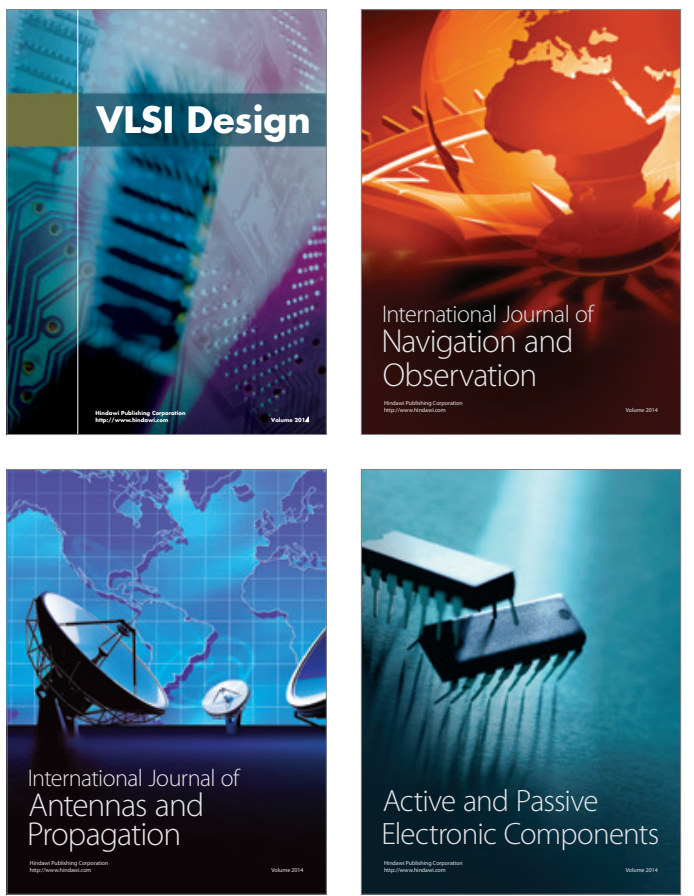
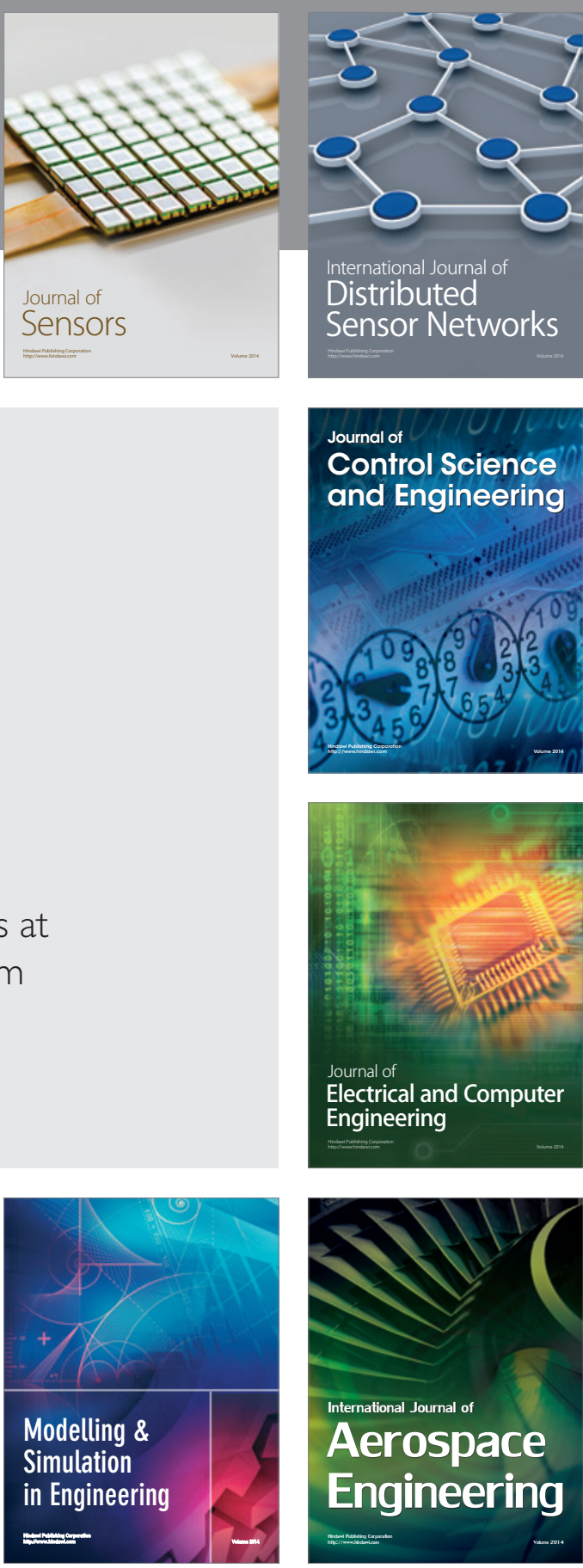

International Journal of

Distributed

Sensor Networks

Journal of

Control Science

and Engineering
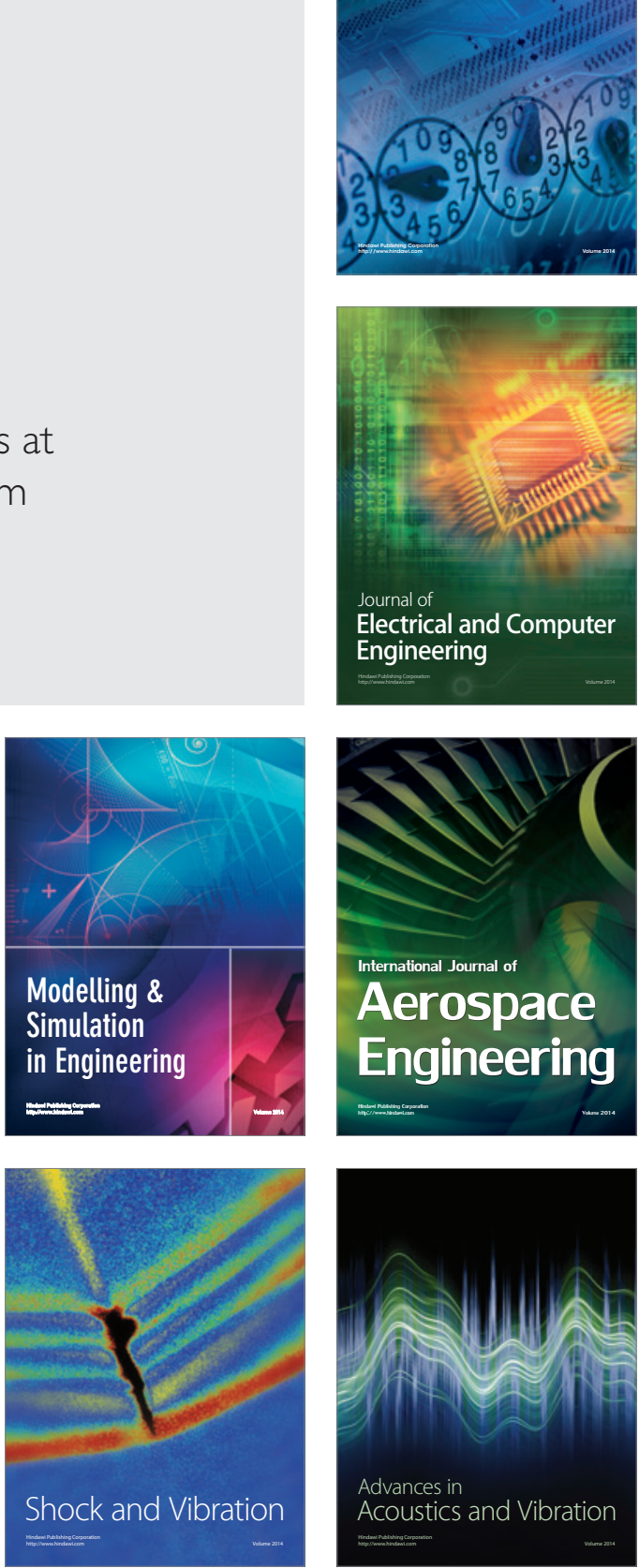